\title{
Neuromyelitis Optica as an Initial Presentation of Systemic Lupus Erythematosus in a Young Male
}

\author{
Sadaf Iftikhar1, Adnan Mahmood ${ }^{1}$ and Masooma Hashmat ${ }^{2}$ \\ 1Department of Neurology, King Edward Medical University / Mayo Hospital, Lahore, Pakistan \\ ${ }^{2}$ Department of Rheumatology, Shaikh Zayed Postgraduate Medical Institute, Lahore, Pakistan
}

\begin{abstract}
Aquaporin-4 antibodies are hallmark of neuromyelitis optica (NMO) and neuromyelitis optica spectrum disorders (NMOSD) that are distinct disease entities. We report a 24-year male with NMO, who presented with Brown-Sequard syndrome. Clinical and laboratory parameters confirmed the diagnosis of systemic lupus erythematosus (SLE). Involvement of sympathetic trunk causing Horner's syndrome and a wide time lapse of 13 years from the occurrence of optic neuritis to myelitis with no relapses in between is a rarity to be seen in NMO associated with SLE.
\end{abstract}

Key Words: Neuromyelitis optica, Systemic lupus erythematosus, Brown-Sequard syndrome, Aquaporin-4 antibodies.

How to cite this article: Iftikhar S, Mahmood A, Hashmat M. Neuromyelitis Optica as an initial presentation of Systemic Lupus Erythematosus in a young male. J Coll Physicians Surg Pak 2020; 30(3):321-323.

\section{INTRODUCTION}

Neuromyelitis optica (NMO), long considered a clinical variant of multiple sclerosis (MS), is now regarded as a distinct disease entity. Major progress has been made in the diagnosis of NMO since aquaporin-4 antibodies (anti-AQP4 Abs; also termed NMO-IgG) were first described in 2004. ${ }^{1}$ The identification of anti-AQP4 antibodies beyond the current diagnostic criteria of NMO indicates a broader clinical phenotype of this disorder, so-called NMO spectrum disorder (NMOSD). The NMOSD encompasses anti-AQP4 antibody seropositive patients with inaugural or limited forms of $\mathrm{NMO}$ and with specific brain abnormalities. It also includes anti-AQP4 antibody seropositive patients with other autoimmune disorders such as systemic lupus erythematosus (SLE) and Sjogren's syndrome (SS). Approximately one-half of NMO patients present with isolated optic neuritis (ON), of which about $20 \%$ are bilateral. Profound and persistent visual loss is a hallmark of ON in NMO, but not in MS. The ON precedes longitudinally extensive transverse myelitis (LETM) in $80 \%$ of cases, usually by less than 3 months. Two-thirds of cases have a relapsing course, and one-third a monophasic illness, most with incomplete recovery and variable persisting disabilities.

We describe a case of young male with $\mathrm{NMO}$ as an initial presentation of SLE who clinically presented with Brown-Sequard syndrome, and Horner's syndrome,

Correspondence to: Dr. Sadaf Iftikhar, Department of

Neurology, King Edward Medical University / Mayo Hospital,

Lahore, Pakistan

E-mail: sadafneurology@kemu.edu.pk

Received: February 06, 2019; Revised: April 09, 2019;

Accepted: April 20, 2019
13 years from the development of $\mathrm{ON}$ at the age of 11 years, with no relapses in between, a rare combination of event in this condition.

\section{CASE REPORT}

A 24-year male presented with acute-onset pain in the nape of his neck, rapidly-progressive weakness of the right side of his body, abnormal sensations on the left side with no bladder bowel involvement. There was history of bilateral ON without recovery at the age of 11 years. On systemic enquiry, there was also history of low-grade fever, off and on, oral ulcers and photo-sensitivity.

On examination, he had malar rash on his cheeks and nose, partial ptosis, miosis and enophthalmos in the right eye and afferent pupillary defect in the left eye (Figure 1). Visual acuity was light perception on the left while finger counting in the right eye. Fundoscopy showed bilateral optic atrophy. He had anhidrosis on his right half of face and upper extremity. Tone was spastic

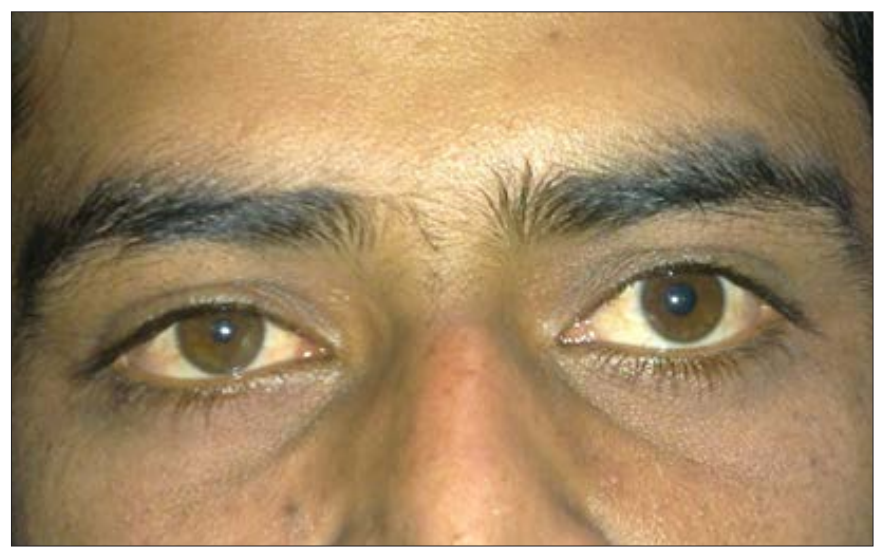

Figure 1: Malar rash and right Horner's syndrome. 


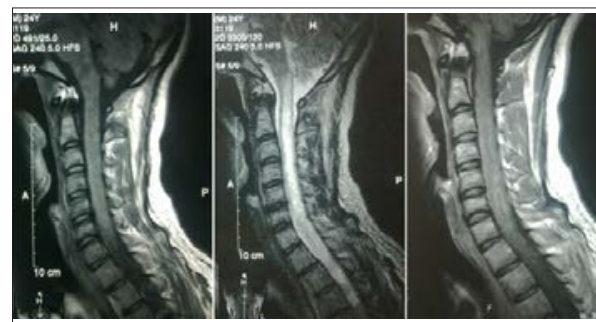

Figure 2: Sagittal T1, T2 and Gadolinium MR imaging showing cord swelling, hyper-intense signal and contrast enhancement in the cervical cord.

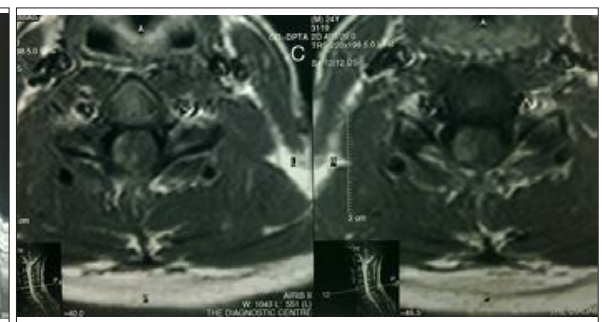

Figure 3: Axial Gadolinium MR imaging showing contrast enhancement in the right hemi-section of cervical cord.

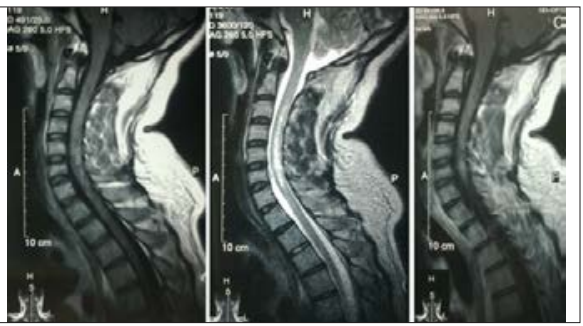

Figure 4: Follow-up, Sagittal, T1, T2 and Gadolinium MR imaging after 4 months showing resolution of swelling, hyper-intense signal and contrast enhancement in the cervical cord. with $3 / 5$ power in the upper and $2 / 5$ in lower extremity with brisk deep tendon reflexes on the right side and normal motor examination on the left side. Plantar response was upgoing on the right side and equivocal on the left side. There was loss of vibration and proprioception up to T2 on the right side and loss of pain and temperature up to the same level on the left side, clinically making the diagnosis of Brown-Sequard syndrome.

His complete blood count showed low white and red blood cells and platelets. Erythrocyte sedimentation rate (ESR) was raised. Urine complete examination showed proteinuria. Anti-nuclear and double stranded DNA antibodies were positive. Cerebrospinal fluid examination showed proteins, $140 \mathrm{mg} / \mathrm{dl}$, glucose, $60 \mathrm{mg} / \mathrm{dl}$, total leukocyte count $32 / \mathrm{cm} 3$ with predominantly lymphocytes. Oligoclonal bands were negative while anti-AQP4 antibodies were positive. Marked retinal nerve fibre layer (RNFL) thinning was seen in both eyes on optical coherence tomography (OCT). Brain magnetic resonance imaging (MRI) with Gadolinium was normal while cervical MRI showed cord swelling on T1W, hyperintensity on T2W and gadolinium enhancement throughout the cervical cord (Figure 2), involving the right side of the cord as shown in axial section (Figure 3 ). Follow-up cervical MRI 4 months later showed marked reduction in the cord swelling, T2W hyperintensity and gadolinium enhancement (Figure 4).

Initially, patient was given pulse methylprednisolone therapy, but no improvement was seen. Five sessions of plasmapheresis were done that significantly improved power to $4+/ 5$ in both upper and lower extremities on the right side. He was then discharged on azathioprine, $50 \mathrm{mg}$, oral once daily, with gradual increments of $50 \mathrm{mg}$ on weekly basis reaching the maintenance dose of $50 \mathrm{mg}$, oral, three times a day. He was also referred for the rheumatological consultation and followed up in neurology outpatient department every 4-6 weeks.

\section{DISCUSSION}

NMO and NMOSD are associated with autoantibodies that target aquaporin-4 and, in many cases, multiple other autoantibodies, including antinuclear antibodies and antibodies to extractable nuclear antigens. The clinical syndromes that define NMO and NMOSD, especially LETM and ON, can also occur in the context of established rheumatologic diseases such as SLE and SS and other organ-specific autoimmune diseases. These observations suggest that NMO is one manifestation of a genetic tendency toward humoral autoimmunity. Alternatively, they might indicate that $\mathrm{NMO}$ is a central nervous system complication of a multisystem rheumatologic disease. 2 NMO and NMOSD has rarely been reported as a first manifestation of SLE. Concerning their association with autoimmune disorders, it is proposed that presence of anti-AQP4 antibodies in patients with SLE and SS is not an epiphenomenon, but rather coexistence of two auto-immune disorders. American College of Rheumatology (ACR) categorised neuropsychiatric manifestations of SLE (NPSLE) into 19 different syndromes. ${ }^{3}$ Pathogenic mechanisms underlying these manifestations are varied and linked to specific clinical manifestations such as small vessel vasculopathy, antibody mediated neurotoxicity, thrombosis of vessels, atherosclerosis, demyelination and inflammatory cytokines causing disruption of blood brain barrier. 4

Our case is distinct in many ways from the previously published case reports, as it is a first case of NMO as an initial presentation of SLE in a young male. All previously reported cases were women of reproductive age group. 5,6 The reasons for the female preponderance of these conditions remain unclear. The most intriguing theory to explain the female predominance is currently related to sex chromosomes, as women with autoimmune diseases manifest a higher rate of circulating cells with a single $X$ chromosome (i.e. $X$ monosomy). In addition, there have been several reports on the role of $X$ chromosome gene dosage through inactivation or duplication in autoimmunity. Taken together, sex chromosome changes might constitute the common trait of the susceptibility to autoimmune diseases. ${ }^{7}$ Men with Klinefelter syndrome $(47, X X Y)$ have the same high prevalence of SLE as women; and have a high prevalence of autoimmune thyroid disease, SS, Addison's syndrome, diabetes, rheumatoid arthritis and MS compared with men without a known chromosomal anomaly. ${ }^{8}$ So, there must be some other mechanisms 
involved in addition to sex chromosome changes in the etiopathogenesis of these autoimmune diseases that are yet to be discovered.

The most distinct manifestation of NMO is LETM, defined as a lesion that spans over three or more contiguous vertebral segments and preferentially involves central gray matter of the spinal cord. This is because aqvaporin-4 is abundant in the gray matter and in glial cell processes adjacent to the ependymal cells of the central canal and to a lesser degree in the white matter of the spinal cord. In contrast, the lesions in MS are mostly localised in the lateral and posterior white matter of the spinal cord. ${ }^{9}$ Our patient clinically presented as Brown-Sequard syndrome and his MRI showed involvement of hemi-section of the cervical cord rather than the central gray matter that is typical of NMO. To our knowledge, this unusual hemi involvement of the spinal cord has not been reported so far in the published data. Also, Horner's syndrome that is observed in our patient, has also not been reported in connection with NMO yet.

Seropositivity for anti-AQP4 antibody is established as a prognostic marker, and its positivity indicates a high risk of further relapses of ON and LETM. ${ }^{10}$ The ON precedes LETM in $80 \%$ of NMO cases, usually by less than 3 months. About $55 \%$ of NMO patients have a relapse in the first year and $90 \%$ in the first five years as per previously published data. In contrast to this, our patient had bilateral $\mathrm{ON}$ at 11 years followed by a wider time interval i.e. 13 years from the occurrence of ON to LETM with no relapses in between, which is quite rarely seen in NMO.

To conclude, a young male presenting with $\mathrm{NMO}$ as an initial presentation of SLE is reported for the first time. Involvement of hemi-section of the cervical cord rather than central gray matter is atypical for NMO and NMOSD. Also, a wide time lapse from ON to LETM with no relapses in between in the presence of seropositivity for NMO-IgG raises concern for the prognostic significance of these antibodies.

\section{PATIENTS' CONSENT:}

Informed consent was taken from the patient for the publication of his image.

\section{CONFLICT OF INTEREST:}

Authors declared no conflict of interest.

\section{AUTHORS' CONTRIBUTION:}

SI: Concept, design, acquisition, drafting and integrity of work.

AM: Acquisition, drafting, analysis and critical appraisal of work

$\mathrm{MH}$ : Acquisition, drafting, analysis and critical appraisal of work

\section{REFERENCES}

1. Lennon VA, Wingerchuk DM, Kryzer TJ, Pittock SJ, Lucchinetti CF, Fujihara K, et al. A serum autoantibody marker of neuromyelitis optica: Distinction from multiple sclerosis. Lancet 2004; 364: 2106-12

2. Asgari N, Jarius S, Laustrup H, Skejoe HP, Lillevang ST, Weinshenker BG, et al. Aquaporin-4-autoimmunity in patients with systemic lupus erythematosus: A predominantly populationbased study. Mult Scler 2018; 24:331-9.

3. The American College of Rheumatology nomenclature and case definitions for neuropsychiatric lupus syndromes. Arth Rheum 1999; 42:599-608.

4. Era MD. Systemic Lupus Erythematosus. In: John BI, David BH, John HS. Current Diagnosis and Treatment Rheumatology. ed. 3rd, McGraw-Hill; 2013.

5. Karim S, Majithia V. Devic's syndrome as initial presentation of systemic lupus erythematosus. Am J Med Sci 2009; 338:245-7.

6. Jacobi C, Stingele K, Kretz R, Hartmann M, StorchHagenlocher B, Breitbart A, et al. Neuromyelitis optica (Devic's syndrome) as first manifestation of systemic lupus erythematosus. Lupus 2006; 15:107-9.

7. Libert C, Dejager L, Pinheiro I. The X chromosome in immune functions: When a chromosome makes the difference. Nat Rev Immunol 2010; 10:594-604.

8. Seminog $O O$, Seminog $A B$, Yeates D. Associations between Klinefelter's syndrome and autoimmune diseases: English national record linkage studies. Autoimmunity 2015; 48:125-8.

9. Kim HJ, Paul F, Lana-Peixoto MA, Tenembaum S, Asgari N, Palace J, et al. MRI characteristics of neuromyelitis optica spectrum disorder: An international update. Neurology 2015; 84:1165-73.

10. Weinshenker BG, Wingerchuk DM, Vukusic S, Linbo L, Pittock SJ, Lucchinetti CF, et al. Neuromyelitis optica IgG predicts relapse after longitudinally extensive transverse myelitis. Ann Neurol 2006; 59:566-9. 